UCRL-JC-121285

PREPRINT

\title{
Increased Damage Thresholds Due to Laser Pulse Modulation
}

\author{
M. D. Feit \\ S. L. Musher \\ A: M. Rubenchik \\ E. G. Shapiro \\ This paper was prepared for submittal to the \\ Solid State Lasers for Application to Inertial \\ Confinement Fusion, Monterey, California \\ May 30-June 2, 1995
}

May 30, 1995

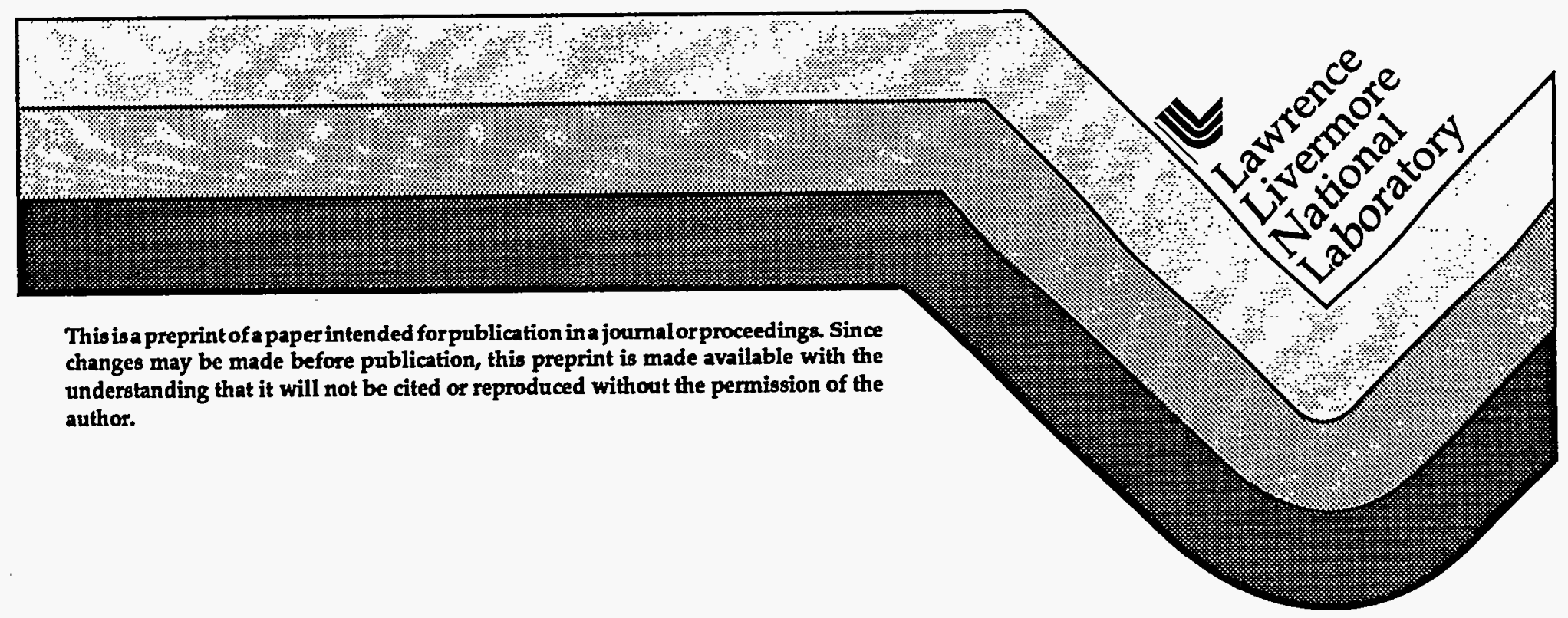




\section{DISCLAIMER}

This document was prepared as an account of work sponsored by an agency of the United States Government. Neither the United States Government nor the University of California nor any of their employees, makes any warranty, express or implied, or assumes any legal liability or responsibility for the accuracy, completeness, or usefulness of any information, apparatus, product, or process disclosed, or represents that its use would not infringe privately owned rights. Reference herein to any specific commercial product, process, or service by trade name, trademark, manufacturer, or otherwise, does not necessarily constitute or imply its endorsement, recommendation, or favoring by the United States Government or the University of California. The views and opinions of authors expressed herein do not necessarily state or reflect those of the United States Government or the University of California, and shall not be used for advertising or product endorsement purposes. 


\section{DISCLAIMER}

Portions of this document may be illegible in electronic image products. Images are produced from the best available original document. 
Solid State Lasers for Application to ICF, Monterey, May 1995

Increased Damage Thresholds due to Laser Pulse Modulation

\author{
M.D. Feit*, S.L. Musher ${ }^{+}$A.M. Rubenchik*t, E.G. Shapiro ${ }^{+}$ \\ * Lawrence Livermore National Laboratory, Livermore, Ca. 94550 \\ + Institute of Automation and Electrometry, Novosibirsk, 630090, Russia
}

Keywords: self-focusing, nonlinear propagation

\begin{abstract}
Nonlinear self-focusing in laser glass imposes limits on the energy fluence that can be safely transmitted without risking damage. For this reason, it is desireable to strictly limit the peak to average spatial variations of fluence by smothing schemes such as Smoothing by Spectral Dispersion (SSD). While spatial variations are problematic, the same is not necessarily true of temporal variations since normal group velocity dispersion tends to smooth out temporal peaks caused by spatial self-focusing. Earlier work [1] indicated that increased bandwidth can delay the onset of self focusing. Indeed, a point can be reached at which self phase modulation nonlinearly increases the bandwidth, changing the speckle statistics along with suppressing self focusing. Unfortunately, this study found that a large initial bandwidth (compared with the gain bandwidth) was necessary to achieve this suppression under practical conditions. The full calculation for modulated beams was carried out for one transverse dimension. Two transverse dimensional calculations only treated symmetric beams. The present work re-examines the question of self focusing threshold increases due to high bandwidth by investigating another source of such increase in three dimensional beam breakup - the bending instability.

For simplicity, we consider the behavior of a single space-time speckle. Normal dispersion can lead to splitting of the pulse [2] and delay of self focusing for short eneough pulses as noted above. In addition to the self focusing instability, the laser beam is also subject to the so-called bending (sausage like) instability which can spatially disperse the field maxima over time [3]. Because the bending instability breaks an initial axial symmetry, a full three dimensional numerical simulation is required to study it accurately. Such calculations are possible, but costly. We have used a modified 2D nonlinear Schrödinger equation with a high power nonlinearity since this mimics the 3D behavior of the competition between self focusing and bending. This allows a semi-quantitative estimate to be made of the possible significance of the bending instability for suppression of self focusing.
\end{abstract}

\title{
Introduction
}

Time averaged smoothing of laser beams by imposition of spectral bandwidth and inducing some form of spatial incoherence is well established. An unfortunate side effect is the lowering of the self focusing threshold due to the presence of intensity maxima. Although the presence of such maxima initially lowers the susceptibility to self focusing, further increases in initial pulse bandwidth can at least partially offset this effect[1] due to group velocity dispersion which tends to reduce temporal maxima. For very large bandwidth, self phase modulation gives further increases in bandwidth and makes group velocity dispersion more efficient. 
Paraxial propagation in a nonlinear dispersive medium can be described by

$$
\mathrm{i} \frac{\partial \mathrm{E}}{\partial \eta}=-\frac{1}{2 \mathrm{~K}}\left(\nabla_{1}^{2} \mathrm{E}+\frac{\omega^{\prime \prime} \mathrm{K}}{\mathrm{v}} \frac{\partial^{2} \mathrm{E}}{\partial \mathrm{Z}^{2}}\right)-\mathrm{Kn}_{2} \mid \mathrm{El}^{2} \mathrm{E}
$$

where $v$ is the group velocity, $\eta=v t$ measures the propagation distance, and $Z=v t-z$ is a retarded time (in spatial units). This equation describes the field in a reference frame that moves with the pulse, ie. at the group velocity. The dispersion coefficient $\omega^{\prime \prime}=\frac{\partial^{2} \omega}{\partial K^{2}}$ can be found from $\left|\frac{\mathrm{v}}{\omega^{\prime \prime} \mathrm{K}}\right|^{1 / 2}=\frac{\partial \ln k}{\partial \ln v}=-\frac{\frac{\lambda^{2} \partial^{2} n}{n} \partial^{2}}{1-\alpha^{2}}$ where $\alpha=\frac{\partial \ln n}{\partial \ln \lambda}$. Typical values for glass are $\alpha=0.015,\left|\frac{v}{\omega " K}\right|^{1 / 2}=5.77$. For normal dispersion, $\omega^{\prime \prime}<0$ and the dispersive term of the propagation equation opposes the diffractive term. Earlier calculations[1] of Eq.(1) with realistic spatial and temporal modulation, but only one transverse dimension indicated that initial bandwidth on the order of $\Delta \omega / \omega=1 \%$ is sufficient to at least significantly delay the onset of self focusing. A few fully three dimensional calculations of single spatial-temporal beampulses did not change this conclusion.

We wish to point out another possible source of suppression of self focusing in normal dispersion glass. For finite size beams, ie. for pulses long compared to transverse dimensions, axial symmetry is broken by the bending instability [3] which reduces the fluence distribution by moving the beam transversely. Because of the lack of symmetry, fully three dimensional numerical calculations are the only way to accurately model this effect. The present work uses a type of nonlinear Schrödinger equation with high power nonlinearity to give a semi-quantitative estimate of the significance of the bending instability with one transverse dimensional calculations.

\section{Theory}

It is convenient to introduce dimensionless variables $x^{\prime}=x / a, z^{\prime}=v t / \mathrm{Ka}^{2}$, $\xi=\left(v / 1 \omega^{\prime \prime} \mathrm{KK}\right)^{1 / 2} \mathrm{Z} / \mathrm{a}, \Psi=\mathrm{Ka} \mathrm{n}_{2}^{1 / 2} \mathrm{E}$ so that the propagation equation reads

$$
\mathrm{i} \frac{\partial \Psi}{\partial z^{1}}=-\frac{1}{2}\left(\nabla 1 \Psi-\frac{\partial^{2} \Psi}{\partial \xi^{2}}\right)-|\Psi|^{2} \Psi
$$

Instead of $\mathrm{Eq}(2)$, we consider the model equation

$$
\mathrm{i} \frac{\partial \Psi}{\partial z}=-\frac{1}{2}\left(\frac{\partial^{2} \Psi}{\partial x^{2}}-\frac{\partial^{2} \Psi}{\partial y^{2}}\right)-|\Psi|^{4} \Psi
$$


Without the negative dispersive term, Eq.(3) is a type of one transverse dimensional nonlinear Schrodinger equation. It is similar to the axisymmetric solution of the two transverse dimensional pure self focusing equation in the intensity dependence on distance to focus.

As in the 2D equation, a nondispersive eigensolution exists of the form

$$
\Psi_{0}=\exp \left(\mathrm{i} \beta^{2} \mathrm{z}\right) \frac{\left(3 \beta^{2}\right)^{1 / 4}}{[\cosh [2 \beta \mathrm{x}]]^{\frac{1}{2}}}
$$

Analogous to the Townes mode, this distribution carries a critical power $P_{c}$. At powers less than critical, the mode diffracts and for powers greater than critical, it self focuses. It can be shown that this mode is an unstable solution of Eq.(3) and gives rise to two types of instability as does the normal 2D nonlinear Schrodinger equation. The first type is symmetric in $x$ and results in self focusing. This mode is similar to the Bespalov-Talanov plane wave instability [4]. For a perturbation of form $\exp (i \Omega y+\gamma z)$, the growth rate, calculated for long wavelength perturbations by the methods in [3] is found to be $\gamma \approx \frac{4}{\sqrt{\pi}} \Omega^{1 / 2} \beta^{3 / 4}$. The second type of instability is asymmetric and leads to beam bending for beams of finite transverse extent. The growth rate for this mode is $\gamma \approx \sqrt{2} \Omega \beta$.

Maximum growth rates for both instabilities occurs at $\Omega \approx \beta$. Both instabilities have similar growth rates and perturbation scales. In order to study the nonlinear competition between the two, we considered as an initial condition, a perturbed mode with spatial size given by Eq.(4). That is, we took

$$
\Psi=\frac{A\left(3 \beta^{2}\right)^{1 / 4} f(\xi)}{\left(\cosh \left[2 \beta\left(x-\varepsilon x_{0}(\xi)\right)\right]\right)^{1 / 2}}
$$

In Eq.(5), $f(x)$ represents the effect of pulse modulation and $\varepsilon$ (small) is a measure of the natural linear bending that arises from one dimensional smoothing by spectral dispersion (SSD).

When a broad bandwidth beam of central wavelength $\lambda$ is incident on a grating with period $d$ at an angle $\theta_{\mathrm{in}}$, Bragg reflection determines the frequency dependent outgoing angle $\theta_{\text {out }}$ as

$\theta_{\text {out }}=\theta_{\text {out }, 0}-\mathrm{m}(\lambda / \mathrm{d}) \Delta \omega / \omega$ where $\sin \left(\theta_{\text {out }, 0}\right)=\sin \left(\theta_{\text {in }}\right)+m(\lambda / d)$ corresponds to the central frequency and $m$ is the diffraction order. In the coordinate system whose propagation direction is along the central frequency wavevector, the original envelope time dependence given by

$$
F(t)=\int G(\Delta \omega) \exp [i \Delta \omega t] d \Delta \omega
$$


is tilted by the frequency dependent phase differences to show up as a transverse modulation. $F(t)$ in Eq. (7) thus becomes

$$
F(t-x / u)=\int G(\Delta \omega) \exp [i \Delta \omega(t-m \lambda K x / \omega d)] d \Delta \omega
$$

where $\mathrm{K}$ is the wavenumber of the light. The temporal structure now shows up transversely. Said differently, frequency shifted components have an offset output angle which is equivalent to a transverse wavenumber proportional to the frequency shift. Eq.(8) describes the beam at the grating. Note that the physical angle is given by the ratio of transverse to longitudinal wavenumbers and thus is given by Eq.(6). Propagation can further modify Eq(8), but the important point is the correlation between temporal and transverse spatial structure.

\section{Results}

Eq.(3) was solved numerically using a split step spectral method.The temporal and spatial scales were chosen to correspond to typical ICF conditions. For a transverse speckle size of $200 \mu \mathrm{m}$, the longitudinal propagation scale is $12 \pi \mathrm{cm}$, and the corresponding temporal scale is about 0.1 ps. For a bandwidth of $1 \%$, the tilt angle in the scaled dimensionless units is about 0.06 The results shown for the model equation correspond to a bandwidth of just under $1 \%$.

Fig.(1) shows the suppression of self focusing[1] for the one transverse dimensional nonlinear Schrödinger equation corresponding to an input intensity of about 4 $\mathrm{GW} / \mathrm{cm}^{2}$ and white noise bandwidths of $0.2 \%$ and $1 \%$. The nonlinear index was taken as $2.7 \times 10^{-7} \mathrm{~cm}^{2} / \mathrm{GW}$.At the lower bandwidth, dispersion only slightly delays the onset of strong self focusing while at the higher bandwidth value, self focusing is prevented over a long distance. The initial effect of low bandwidth modulation is deleterious compared to the cw case since, by definition, modulation introduces higher than average intensity peaks. Additional bandwidth can then be advantageous since it increases the strength of dispersion. Unfortunately, as noted above, the required bandwidth is impractically large compared to the gain bandwidth.

The quantitative relationship between self focusing and bending can only be determined by numerical simulation. In order to demonstrate suppression of self focusing, we first consider an artificial case in which there is bending but no initial temporal modulation. We take as the initial condition, the perturbed Townes-like modeshape of Eq. (5) with $A=1.32, \beta=1, f(\xi) \equiv 1, \varepsilon=0.1$, and the bending is given by $x_{0}(\xi)=\cos (\xi)$. The calculation was carried out with periodic boundary conditions. The amplitude was chosen to be over the self focusing threshold so that catastrophic focusing occurs in the absence of bending. Fig.(2) shows the evolution of intensity over space and time as the normalized propagation distance increases to 3 . The amplitude of bending increases strongly with distance. The effect of this is to reduce the average intensity or fluence peaks. The fluence curves in Fig.(3) correspond to the intensities in Fig.(2); the reduced values indicate a reduced susceptibility to damage..

Figs. (4) and (5) show typical development of the solution of Eq.(3) with initial field of the form of Eq.(5). The figures show the field amplitude as a function of scaled transverse $(x)$ and longitudinal $(\xi)$ coordinates at distances of $z=0.28$ and 0.294 . The later 
corresponds to a B integral of just under 2. The physical tilt angle in this case is $8.3 \mathrm{mrad}$ and the bandwidth is $1 \%$. The modulation function $f(\xi)$ in Eq. (5) was taken as a power 6 superGaussian function of width 3.5 so the pulse length is $0.8 \mathrm{ps}$. The figures show the typical strong self focusing behavior. The intensity is growing rapidly and dispersion is incapable of arresting this growth.

Of course, dispersion is sensitive to the curvature of the pulse at the peak intensity. If the superGaussian shape is replaced by an equivalent Gaussian, self focusing is much less severe as shown in Fig.(G) which refers to a B integral of 2.4 . The difference in these two cases may be due, in part, to the increased bandwidth of the Gaussian pulseshape.

\section{Conclusion}

We have found that the threshold of self focusing is decreased for low bandwidth modulation as stated in [1] and that further increases in bandwidth then increase the threshold because of group velocity dispersion. Preliminary calculations with the model equation indicate that the bending instability, which also tends to limit self focusing by spreading the beam transversely in time, has a similarly sized influence on self focusing.This is reasonable since, at a given transverse position, bending appears as temporal modulation of the pulse. Thus, both deliberate temporal modulation and bending can be sources of increased bandwidth. Full three dimensional self focusing can be limited by high enough bandwidth such that dispersion takes place before strong self focusing. The required bandwidth, for typical ICF conditions, is on the order of $1 \%$ which is rather large in comparison to the gain bandwidth.

Since the bending instability reduces the peak fluence (or time averaged intensity), it has a beneficial effect on the onset of damage. The results reported here are relevant to recent observations [5] that the damage induced by short (sub-picosecond) high bandwidth pulses has a threshold determined largely by fluence. Such ultrashort pulses can have intensity high enough to cause self focusing with fluence below the damage threshold.

\section{Acknowledgement}

Work of MDF and AMR was performed under the auspices of the U.S. Department of Energy by Lawrence Livermore National Laboratory under contract No. W-7405-ENG-48.

\section{References:}

1. M.D. Feit and J.A. Fleck, Jr., "Self-focusing of broadband laser pulses in dispersive media", LLNL report UCRL-ID-112523 (Dec. 1992). See also paper P19 at this conference : "Measurement of the Bespalov-Talanov gain spectrum in a dispersive medium with large n2", P. Wegner, M.D. Feit, J.A. Fleck,D. Eimerl and H.T. Powell

2. J.E. Rothenberg,"'Pulse Splitting During Self-Focusing In Normally Dispersive Media"; Optics Letters 17, 583 (1992)

3. E. Kuznetsov, A.M. Rubenchik, and V. Zakharov, "Soliton stability in plasmas and hydrodynamics", Phys. Rpts. 142, 103, (1986)

4.V.I. Bespalov and V.I. Talanov, JETP Letters 3, 307 (1966)

5. B.C. Stuart, M.D. Feit, A.M. Rubenchik, B.W. Shore and M.D. Perry, "Laser induced damage in dielectrics with nanosecond to subpicosecond pulses", Phys. Rev. Lett 74, 2248-51 (March 1995) 


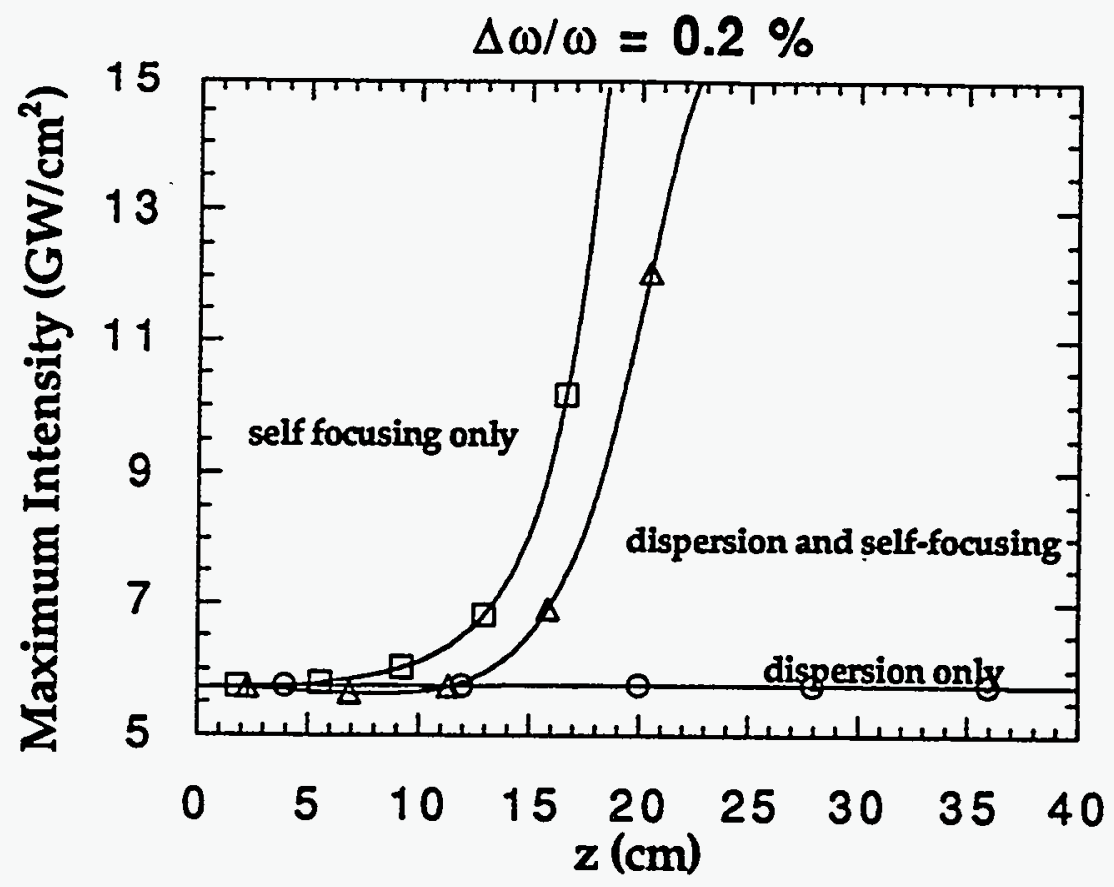

Fig. (1a) Development of maximum intensity for amplitude modulated pulses in one transverse dimension. For bandwidth $\ll<1 \%$, group velocity dispersion can only slightly delay the onset of self focusing.

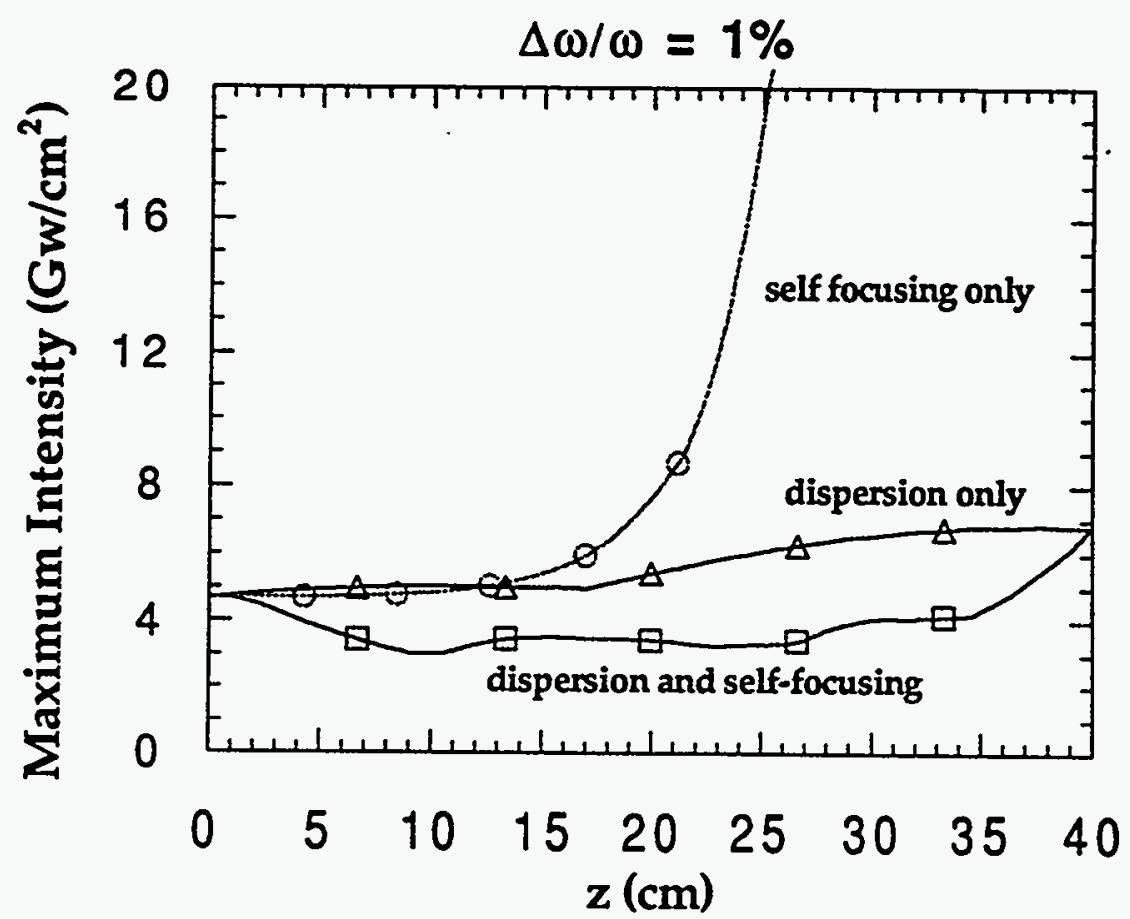

Fig.(1b): Development of maximum intensity for amplitude modulated pulses in one transverse dimension. At bandwidth of $1 \%$, group velocity dispersion arrests self focusing. Note markers only used to distinguish curves. 

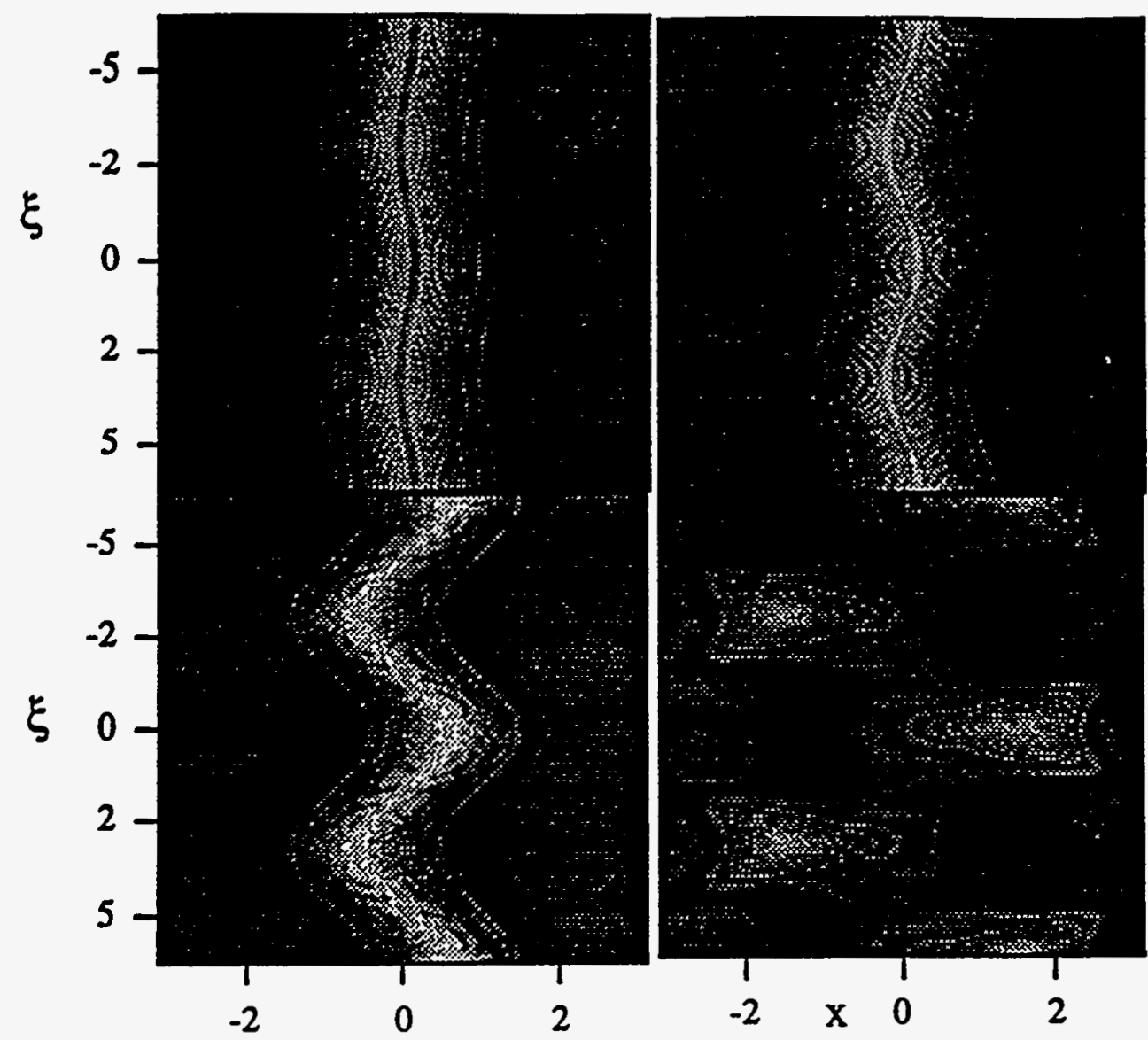

\section{Intensity}

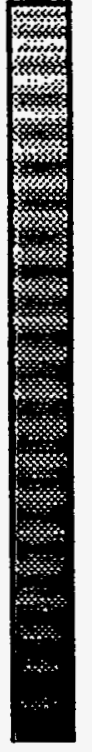

Fig. 2: Evolution of intensity for beam with initial bending and no temporal modulation. Initial intensity just over self focusing threshold. Pictures correspond to distances of $\mathrm{z}=0,1,2,3$. 


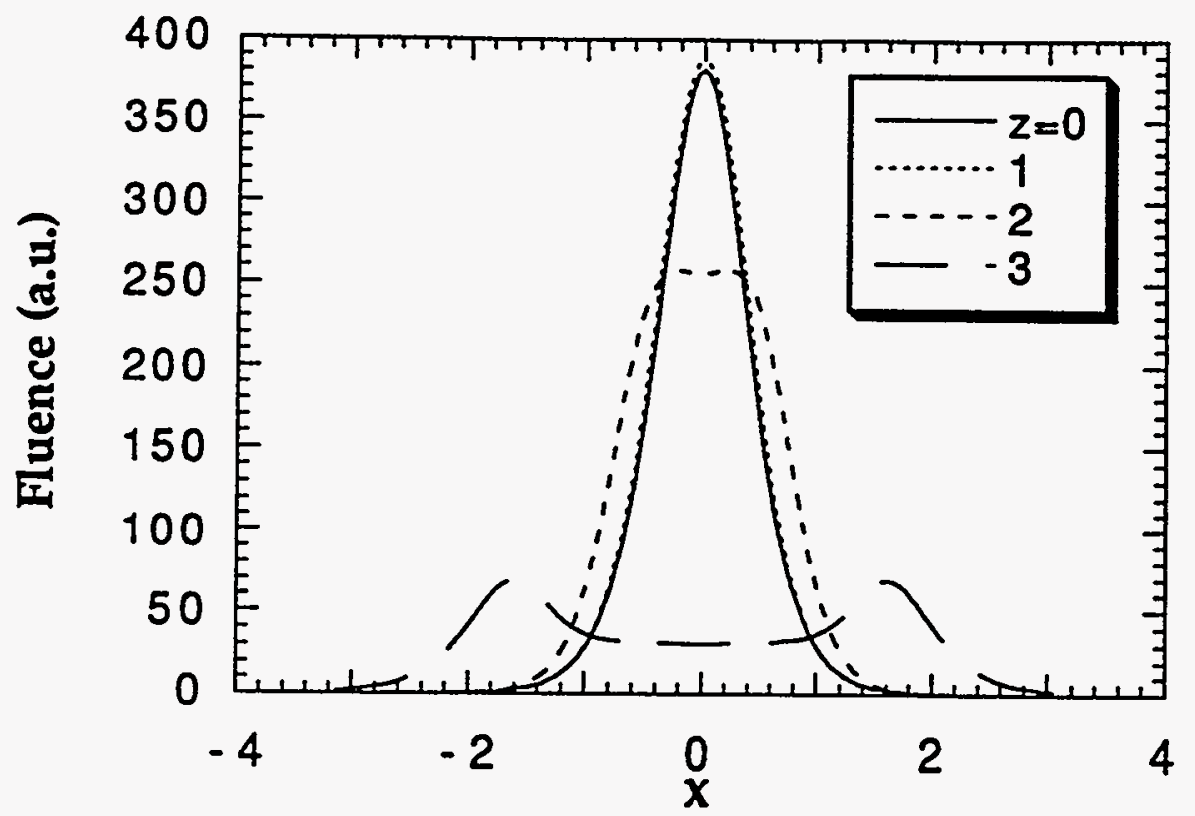

Fig. 3: Reduction in peak fluence caused by growth of bending accompanying nonlinear propagation. Curves correspond to intensities shown in Fig.(2) at distances of $z=0,1,2,3$.

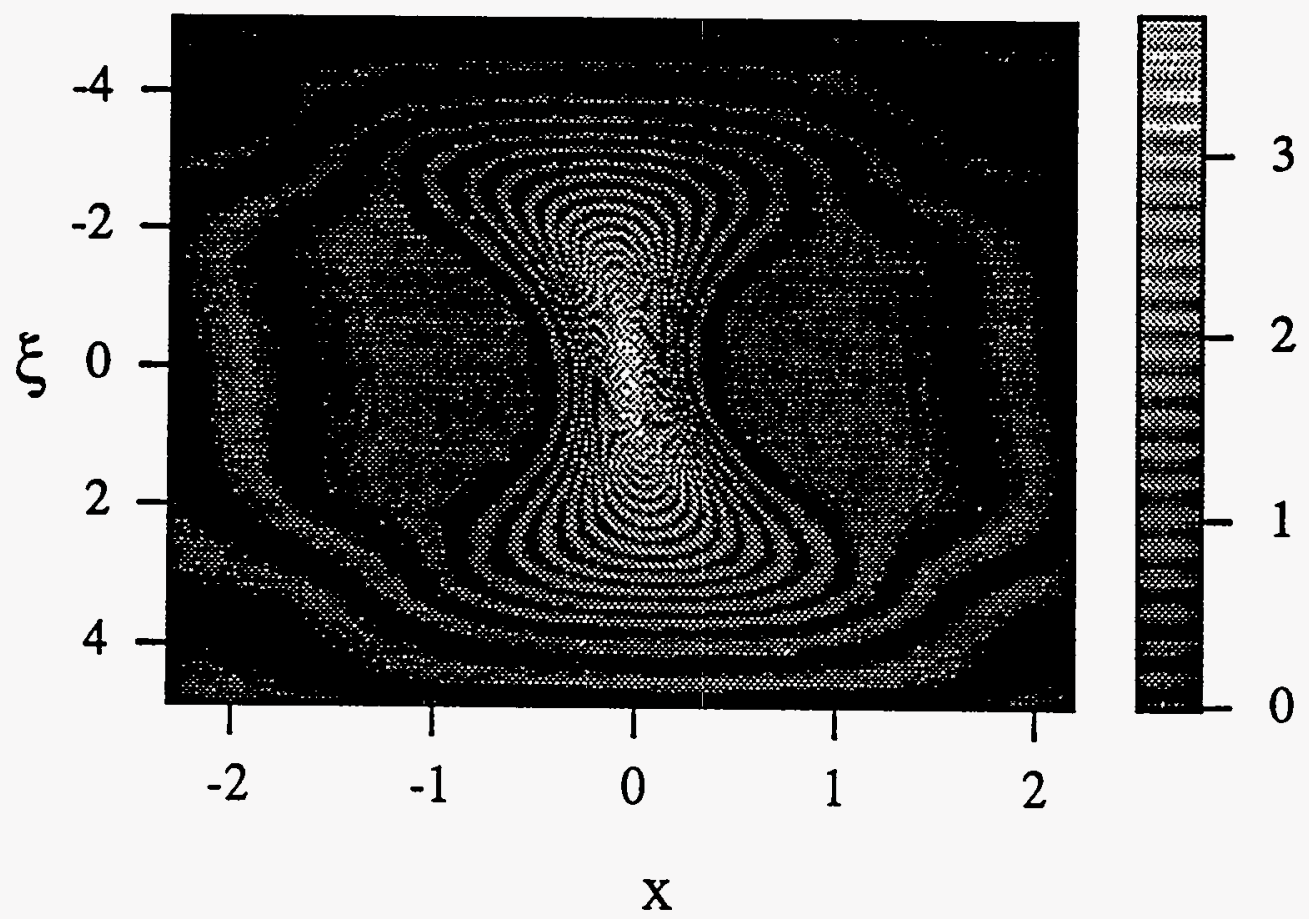

Fig. 4: Distribution of amplitude of model equation at normalized distance $z=0.28$. Initial conditions correspond to $1.5 \mathrm{GW} / \mathrm{cm}^{2}$ superGaussian pulse with length of $0.8 \mathrm{ps}$. Physical tilt angle is $8 \mathrm{mrad}$. Narrowing at peak is demonstrates beginning of self focusing. 